\title{
Editorial: Green light for Animal Genetic Resources
}

Global recognition of the essential contribution of animal genetic resources to food security, sustainable rural development, and management of natural resources has led to the initiation of the first global assessment of the state of these resources. The initiative was taken in 1999 by the FAO Commission on Genetic Resources for Food and Agriculture, which has 161 members and is the major international forum for developing policies on genetic resources for food and agriculture. The Commission requested that FAO should co-ordinate the development of a country-driven Report on the State of the World's Animal Genetic Resources. It noted the need for guidelines to assist country participation in the preparation of the Report. The Commission also requested that the Intergovernmental Technical Working Group on Animal Genetic Resources review the guidelines which was done at its second session on 4-6 September 2000. This session also established the timeline for countries to prepare their Reports.

Countries will be invited by FAO to prepare their Country Reports using the approved guidelines. The Country Report will be an official government document that provides a strategic assessment of the state of animal genetic resources and the capacity to manage them. National Consultative Committees will be formed to oversee preparation of the Country Reports. These bodies will include representatives from the entire range of interests in animal genetic resources, including government agencies, industry, non-government organizations, and local communities. Networks should be established covering the range of important animal species and production systems. National Committees will ensure that their Country Reports address three fundamental strategic questions: Where is the country in terms of the management of animal genetic resources? Where does the country need to be in terms of the management of these resources over the next 5 to 10 years? How does the country get to where it needs to be? The most important objective of the Country Report is to provide a strategic assessment of the country's animal genetic resources sector, so that it enables development of a strategic plan for animal genetic resources management.

Country Reports will be based on collation and analysis of existing data and information on animal genetic resources within their relevant production systems, to support the strategic analysis. This will include data and information on the state of understanding of the diversity of the countries' animal genetic resources, the state of capacity to manage them, and data and information to assist the National Committee to predict future country needs.

Country and regional networking will be essential to assemble the best available data and information. Also, networking will help stimulate greater awareness of the many roles and values of these essential resources and gain appreciation of the need for investments in their wise use and development.

Investments are also required in conservation to meet the many challenges posed by changing consumer demands and environmental conditions.

Regional networks are essential to facilitate the identification of strategic priorities for co-operation and collaboration among countries with common or shared animal genetic resources, and those with common production systems and/or issues.

FAO will provide a supporting and co-ordination role, by providing guidelines, training, planning and monitoring, data collection and analysis, and Country Report 
tools within DAD-IS, providing on-line support to National Committees, and by providing regional training workshops.

Country Reports are to be completed by the end of 2001 to enable the synthesis of the report at the global level, as well as to incorporate data, information, and strategic priorities provided from relevant international organizations. The first product of the synthesis, the Report on Strategic Priority Actions will be presented to the Commission in 2003. The first Report on the State of the World's Animal Genetic Resources and its follow-up mechanism will be presented to the Commission in 2005.

The challenging and demanding efforts to prepare Country Reports will provide an essential foundation for the better management of animal genetic resources.
These resources are rapidly eroding and it is clear that in many countries, locally adapted breeds are not being utilized or developed to their potential. Faced with food shortages, many countries feel forced to import exotic animal genetic resources without either the tools or capacity to properly assess their appropriate use and long-term impacts, or to consider how best to use their indigenous resources. In many countries, the Reports will provide for the first time a strategic basis to assess and plan the better management of animal genetic resources. Country Reports and the first Report on the State of the World's Animal Genetic Resources will provide a strong signal for enhanced regional and global cooperation to achieve the better use of animal genetic resources and to prevent their indiscriminate use.

The Editors 


\section{Editorial: Feu vert pour les Ressources Génétiques Animales}

La reconnaissance mondiale de la contribution essentielle des ressources génétiques animales à la sécurité alimentaire, au développement rural durable et à la gestion des ressources naturelles a porté à la première évaluation mondiale sur la situation de ces ressources. L'initiative avait été prise en 1999 par la Commission pour les Ressources Génétiques pour l'Alimentation et l'Agriculture de la FAO, qui compte 161 membres et est le forum international le plus important pour le développement des politiques des ressources génétiques pour l'alimentation et l'agriculture. La Commission a sollicité à la FAO de coordonner le développement d'un rapport à base nationale sur La Situation des Ressources Génétques dans le Monde. La Commission a aussi noter le besoin de lignes guide pour aider la participation des pays à la préparation de ce rapport. La Commission a demandé également que le Groupe Technique Inter Gouvernemental de Travail pour les Ressources Génétiques Animales revoit les lignes guides qui avaient été préparées lors de sa deuxième session du 4-6 septembre 2000. Cette session a par ailleurs établi les dates limites pour les pays pour la preparation de leurs rapports.

Les pays seront invités par la FAO à préparer leur Rapport National en utilisant les lignes guides approuvées. Le Rapport National sera un document gouvernemental officiel qui fournira une évaluation stratégique sur la situation des ressources génétiques animales et la capacité existante pour leur gestion. Les Comités de Consultation seront formés sur la base des Rapports Nationaux présentés. Ces Comités inclueront des représentants venant de tous les domaines d'intérêt pour les ressources génétiques animales, y compris les agences gouvernementales, l'industrie, les organisations non gouvernementales et les communautés locales. Des réseaux seront établis pour couvrir toutes les espèces animales et les systèmes de productions plus importants. Les Comités Nationaux assureront que leur Rapport National tient compte de trois questions stratégiques fondamentales: A quel point se trouve le pays en termes de gestion des ressources génétiques animales? A quel niveau pensent-ils se trouver dans 5 à 10 ans en termes de gestion des ressources génétiques animales? Que pense faire le pays pour atteindre son but? L'objectif le plus important du Rapport National est de fournir une évaluation stratégique du secteur national des ressources génétiques animales, de façon à permettre le développement d'un plan stratégique de gestion de ces ressources.

Les Rapports Nationaux seront basés sur la collation et l'analyse des données et

l'information existantes sur les ressources génétiques animales dans le contexte de leurs systèmes de production afin d'aider l'analyse stratégique. Ceci devra inclure des données et l'information sur la situation de sensibilisation de la diversité des ressources génétiques animales dans le pays, la capacité de gestion de ces ressources, et les données et information nécessaires pour aider le Comité National à prévoir les besoins futurs du pays.

Les réseaux nationaux et régionaux seront essentiels pour rassembler les données et l'information disponibles plus intéressantes. En outre, les réseaux aideront à stimuler une plus grande concience des différents rôles et valeurs de ces ressources essentielles ainsi qu'à augmenter la nécessité du besoin d'investissements pour leur plus large utilisation et développement. Les investissements seront aussi nécessaires pour la conservation afin de faire face aux différents défis posés par les changements dans la demande des consommateurs et des conditions environnementales. 
Les réseaux régionaux sont essentiels pour faciliter l'identification des priorités stratégiques pour la coopération et la collaboration entre les pays pour les ressources génétiques animales communes ou partagées, ainsi que ceux avec des systèmes de production et/ou des débouchées communs.

La FAO aura un rôle d'appui et de coordination et fournira donc les lignes guides, la formation, la planification et suivi, la saisie et l'analyse de données, et les outils de DAD-IS pour le Rapport National, à travers une aide on line aux Comités Nationaux et l'organisation d'ateliers de formation au niveau régional.

Les Rapports Nationaux devront être complèté avant la fin de 2001 pour permettre la synthèse du rapport au niveau mondial ainsi que l'introduction de données, d'information et de priorités stratégiques fournis par les organisations internationales pertinentes. Le premier résultat de synthèse, le Rapport sur les Actions Stratégiques Prioritaires, sera présenté à la Commission en 2003. Le premier Rapport sur la Situation Mondiale des Ressources Génétiques Animales et son mécanisme de suivi sera présenté à la Commission en 2005.
Les efforts de stimulation et de demande nécessaires pour préparer les Rapports Nationaux fourniront une base essentielle pour une meilleure gestion des ressources génétiques animales. Ces ressources souffrent d'une érosion rapide et il est clair que dans beaucoup de pays les races adaptées aux conditions locales ne sont pas utilisées ou développées aux limites de leur potentiel. Face au déficit alimentaire, beaucoup de pays se sentent obligés à importer des ressources génétiques exotiques sans avoir pour autant les outils ou la capacité pour évaluer correctement leur utilisation exacte et l'impact possible à long terme, ou bien pour considérer comment mieux utiliser leurs ressources autochtones. Dans beaucoup de pays les Rapports fourniront pour la première fois les bases stratégiques pour évaluer et planifier une meilleure gestion des ressources génétiques animales. Les Rapports Nationaux et le premier Rapport sur la Situation Mondiale des Ressources Génétiques Animales lanceront un important signal d'encouragement pour une coopération régionale et mondiale à atteindre une meilleure utilisation des ressources génétiques animales et à prévenir leur utilisation indiscriminée. 


\section{Editorial: Luz Verde para los Recursos Genéticos Animales}

El reconocimiento mundial de la contribución esencial de los recursos genéticos animales a la seguridad alimentaria, al desarrollo rural sostenible y a la gestión de los recursos naturales ha llevado a la primera evaluación mundial sobre la situación de estos recursos. La iniciativo fue tomada en 1999 por la Comisión para los Recursos Genéticos para la Alimentación y la Agricultura de la FAO, que cuenta con 161 miembros y es el forum internacional más importante para el desarrollo de políticas sobre recursos genéticos para la alimentación y la agricultura. La Comisión solicitó a la FAO que coordinase el desarrollo de un informe sobre base nacional sobre La Situación de los Recursos Genéticos en el Mundo. La Comisión también subrayó la necesidad de establecer directrices para fomentar la participación de los países en la preparación de dicho informe. La Comisión pidió también que el Grupo Técnico Inter Gubernamental de Trabajo para los Recursos Genéticos Animales revisara las directrices que habían sido preparadas durante su segunda sesión del 406 septiembre 2000. Por otra part, esta sesión estableció las fechas límite para la preparación de los informes por parte de los países.

La FAO invitará los países a preparar su Informe Nacional utilizando las directrices aprobadas. El Informe Nacional será un documento gubernamental oficial que proveerá una evaluación estratégica sobre la situación de los recursos genéticos animales y la capacidad existente para su gestión. Los Comités de Consulta se formarán en base a los Informes Nacionales presentados. Estos Comités incluirán representantes provenientes de todos los sectores di interés para los recursos genéticos animales, incluidas las agencias gubernamentales, la industria, las organizaciones non gubernamentales y las comunidad locales. Se establecerán redes para cubrir todas las especies animales y los sistemas de producción más importantes. Los Comités Nacionales se aseguraran que sus Informes Nacionales tienen en cuenta tres cuestiones estratégicas fundamentales: A que punto se encuetra el país en términos de gestión de los recursos genéticos animales? Que nivel piensan poder alcanzar en un plazo de 5 a 10 años en cuestión de gestión de los recursos genéticos animales? Que piensa hacer el país para alcanzar su objetivo? El objetivo más importante del Informe Nacional es proveer una evaluación estratégica del sector nacional de los recursos genéticos animales, para permitir el desarrollo de un plan estratégico de gestión de estos recursos.

Los Informes Nacionales se basarán en la comparación y el análisis de datos e información existentes sobre los recursos genéticos animales dentro del contexto de sus sistemas de producción, con el fin de facilitar el análisis estratégico. Para ello, se incluirán los datos y la información sobre el estado de sensibilización frente a la diversidad de los recursos genéticos animales en el país, la capacidad de gestión de estos recursos, y los datos e información necesarios para ayudar al Comité Nacional en su previsión de las necesidades futuras del país.

Las redes nacionales y regionales serán esenciales para recoger los datos y la información más interesante disponibles. Además, las redes ayudarán a estimular una mayor conciencia sobre los distintos papeles y valores que representan estos recursos esenciales, así como a aumentar la necesidad de inversión para una mayor utilización y desarrollo de los mismos. Las inversiones serán también necesarias para la conservación, para poder enfrentarse a los distintos desafíos planteados por los cambios de tendencia en la demanda de los consumidores y por las condiciones ambientales. 
Las redes regionales son esenciales para facilitar la identificación de prioridades estratégicas para la cooperación y la colaboración entre países con recursos genéticos animales comunes o compartidos, así como para aquéllos con sistemas de producción y/o salidas comunes.

La FAO jugará un papel de apoyo y coordinación y proveerá las directrices, la formación, la planificación y seguimiento, la introducción y el análisis de datos, así cmo las herramientas de DAD-IS para el Informe Nacional, a través de una ayuda on line a los Comités Nacionales y la organización de talleres de formación a nivel regional.

Los Informes Nacionales deberán ser completados antes de finales del 2001 para permitir una síntesis del informe a nivel mundial así como la introducción de datos, información y prioridades estratégicas remitidos por las organizaciones internacionales pertinentes. El primer resultado de síntesis, el Informe sobre la Situación Mundial de los Recursos Genéticos Animales y su mecanismo de seguimiento, será presentado a la Comisión en el 2005.

Los desafíos y las solicitudes a las que habrá que enfrentarse para preparar los
Informes Nacionales proporcionarán una base esencial para una mejor gestión de los recursos genéticos animales. Estos recursos padecen de una erosión rápida y está claro que en muchos países las razas adaptadas a las condiciones locales no vienen utilizadas o desarrolladas hasta el límite de su potencialidad. Frente al deficit alimentario, muchos países se sienten obligados a importar recursos genéticos exóticos sin poseer las herramientas necesarias o la capacidad para evaluar correctamente el uso exacto y el posible impacto a largo plazo, como tampoco saben cómo utilizar mejor sus propios recursos autóctonos. En muchos países los Informes permitirán por primera vez contar con bases estratégicas para evaluar y planificar una mejor gestión de los recursos genéticos animales. Los Informes Nacionales y el primer Informe sobre la Situación Mundial de los Recursos Genéticos Animales lanzarán un importante señal para fomentar una mayor cooperación regional y mundial y obtener así una mejor utilización de los recursos genéticos animales así como una prevención del uso indiscriminado. 